\title{
Panniculus carnosus, Remanentes Vestigiales en la Región Axilar
}

\author{
Panniculus carnosus, Vestigial Remanents in the Axillary Region \\ Oscar Inzunza; Arnaldo Marín; Felipe Pino; Cristian Navarrete \& Alex Vargas
}

INZUNZA, O.; MARÍN, A.; PINO, F.; NAVARRETE, C. \& VARGAS, A. Panniculus carnosus, remanentes vestigiales en la región axilar. Int. J. Morphol., 26(4):841-844, 2008.

RESUMEN: Las variaciones anatómicas de los elementos musculares de la región axilar son poco frecuentes y, generalmente, se encuentran olvidadas en los textos modernos de anatomía. Sin embargo, cuando existen, presentan un serio escollo para el cirujano, ya que alteran los hitos anatómicos de referencia para el abordaje de los importantes elementos vasculares y neurales de la región. Desde el siglo XIX algunos autores han descrito una variedad de pequeños fascículos musculares originados en el Panniculus carnosus, que se disponen en relación con los elementos neurovasculares de la axila. En esta ocasión presentamos un hallazgo efectuado en un cadáver, de sexo masculino, utilizado con fines docentes en el Departamento de Anatomía de la Escuela de Medicina de la Pontificia Universidad Católica de Chile. En la región axilar derecha, se observa un fascículo muscular que se extiende desde la apófisis coracoides, dispuesto por delante del músculo subescapular y pasando por detrás del paquete neurovascular de la axila. En su trayecto, este fascículo toma íntima relación con el nervio axilar y con la porción distal de la arteria subescapular. El músculo aberrante termina como una expansión aponeurótica, dispuesta ventral al tendón del latísimo del dorso, que se funde con la fascia axilar.

PALABRAS CLAVE: Anatomía; Región axilar; Panniculus carnosus.

\section{INTRODUCCIÓN}

Desde fines del siglo XVIII, se han descrito en la fosa axilar bandas o haces de tejido muscular y tendinoso que se disponen entre las paredes de la región (Ramsay, 1795, 1812; Gruber, 1844; Langer, 1846; Wood, 1868); elementos que corresponden a remantes del Panniculus carnosus de los mamíferos inferiores (Langworthy, 1932), que por su situación pueden entorpecer el examen clínico, generar fenómenos compresivos en los vasos axilares, dificultar las acciones quirúrgicas en la región y confundir a los anatomistas (Tillaux, 1880; Petrasek et al., 1997; Merida-Velasco et al., 2003; del Sol \& Olave, 2005).

Sólo en la clase mamíferos se describe este plano muscular, considerablemente desarrollado en algunas especies como los roedores, que se extiende ampliamente sobre el tórax, el abdomen y el dorso; capa muscular de ubicación subcutánea, conocido como Panniculus carnosus (Langworthy, 1924). En humanos y primates este plano muscular involuciona concomitantemente con la mayor movilidad que adquiere el miembro superior, lo que reduce su importancia funcional (Bergman et al., 2006), persistiendo como una estructura anatómicamente evidente sólo a nivel del músculo dártos (Besana-Ciani \& Greenall, 2005).

La situación subcutánea de este plano muscular es adquirida secundariamente, ya que, en realidad, corresponde a musculatura esquelética, en especial musculatura pectoral, que toma relación con la piel (Ruge, 1895) y que se encuentra inervada por ramos primarios ventrales de los nervios torácicos (Langworthy, 1924).

Si bien la literatura especializada señala que la frecuencia de aparición de elementos vestigiales del Panniculus carnosus en la axila es baja -entre 4\% y 12\% según Miguel et al. (2001)-, este dato puede estar subvalorado debido a que fascículos pequeños pueden pasar desapercibidos para el operador inexperto -cirujano o anatomista- (Tillaux; Kutiyanawala et al., 1998). Los datos publicados señalan que estas variaciones musculares son más comunes en mujeres, observándose con mayor prevalencia en mongoloides y caucasoides (Besana-Ciani $\&$ Greenall). Así mismo, se describe una gran variedad de presentaciones, con fascículos músculo-tendinosos originados en el tendón del M. latísimo del dorso y que se extienden hacia el M. pectoral mayor (Ramsay, 1795, 1812; Langer), el M. coracobraquial (Kutiyanawala et al.) o el proceso coracoides (del Sol \& Olave).

Presentamos un hallazgo observado en una de las disecciones de carácter docente, realizada en el Departamento de Anatomía de la Escuela de Medicina de la Pontificia Universidad Católica de Chile; un fascículo muscular y tendinoso extendido entre el proceso coracoides y el tendón del M. latísimo 
INZUNZA, O.; MARÍN, A.; PINO, F.; NAVARRETE, C. \& VARGAS, A. Panniculus carnosus, remanentes vestigiales en la región axilar. Int. J. Morphol., 26(4):841-844, 2008.

del dorso. El análisis de estas variaciones anatómicas, observadas en cadáveres utilizados con fines docentes, es especialmente atractivo para los alumnos y para los docentes, motivándonos a darlas a conocer en múltiples oportunidades. Esto permite resaltar lo formativo de la disección anatómica, método no superado para aprender, realmente, anatomía humana (Bravo \& Inzunza, 1995). Además, estas variaciones constituyen un desafío para los alumnos de pregrado, permitiéndoles aplicar, en este caso, sus conocimientos de anatomía comparada para explicar su génesis.

\section{MATERIAL Y MÉTODO}

En un cadáver de sexo masculino, de 63 años de edad, cuya causa de muerte fue caquexia por tuberculosis pulmonar avanzada que terminó con paro cardíaco, utilizado para fines docentes en el Departamento de Anatomía de la Escuela de Medicina de la Pontificia Universidad Católica de Chile, se realizó una disección de la región axilar derecha. Se identificaron los planos musculares de la pared anterior, para luego identificar la vena y la arteria axilar. En relación a esta última, se reconocen los ramos de los fascículos lateral y medial del plexo braquial. Al continuar la disección, por detrás de la arteria axilar, en la búsqueda de los ramos del fascículo posterior, se identifica un elemento músculo-tendinoso aberrante que se extiende desde la base del proceso coracoides, por delante del M. subescapular, y que termina como una expansión aponeurótica delante del M. latísimo del dorso. Con piedemetro se procedió a mensurar las dimensiones del fascículo muscular; asimismo, se procedió a disecar los elementos neuro-vasculares próximos a él.

\section{RELATO DE CASO}

El fascículo muscular aberrante presentaba un vientre superior, un tendón intermedio y una porción inferior músculo-tendinosa, adoptando la forma de un lente bicóncavo, alargado en el eje próximo-distal, con una longitud total de 112,3 $\mathrm{mm}$. Se extendía desde la base del proceso coracoides hasta la fascia de la base de la axila, tomando inserción en la cara anterior de la porción superior del tendón del M. latísimo del dorso. Las dimensiones de los segmentos eran: Vientre superior: 58,6 mm de largo y 12,5 mm de ancho; Tendón intermedio: 21,3 mm de largo y 2,9 mm de ancho y Porción inferior: $32,4 \mathrm{~mm}$ de largo y $65,5 \mathrm{~mm}$ de ancho; medidos a nivel del borde inferior del tendón del M. latísimo del dorso (Fig. 1).

El vientre superior se disponía sobre la porción lateral del músculo subescapular, tomando relación con una fina rama arterial originada desde la segunda porción de la arteria axilar, que lo penetraba en el punto cercano a su origen, en la base del proceso coracoides. En la cara anterior de este vientre mus- cular una fina rama del nervio pectoral lateral se introducía en la porción carnosa del músculo.

El tendón intermedio surgía por el borde medial del vientre superior, estando cubierto por el paquete neuro-vascular de la axila; dispuesto inmediatamente lateral al nervio axilar y ventral a la porción terminal de la arteria subescapular, su trayecto ocultaba el punto de bifurcación de este vaso en las ramas circunfleja escapular y tóraco-dorsal (Fig. 1).

La porción inferior del fascículo muscular aberrante se ubicaba inmediatamente ventral al tendón del músculo latísimo del dorso, fijándose en él. De aspecto músculo-tendinosa, esta estructura se ensanchaba como pata de anserino, insertándose en el aspecto superior de la fascia axilar. La zona media de esta porción se encontraba reforzada por una extensión tendinosa, que seguía en el eje del tendón intermedio, cruzando de dorsal hacia ventral la fascia de la base de la axila (Fig. 2).

\section{DISCUSIÓN}

Desde hace largo tiempo que en la literatura especializada se han descrito variaciones musculares en la fosa axilar, elementos que se disponen entre sus paredes musculares (Ramsay, 1812; Gruber). Estas variaciones indefectiblemente involucran al tendón del M. latísimo del dorso, en el cual se insertan estas fibras musculares aberrantes, pero cuyo punto de origen puede ser muy variado; por ejemplo: el M. pectoral mayor (Langer, 1846), el M. coracobraquial (Kutiyanawala et al.), el M. serrato anterior (Miguel et al.) o el proceso coracoides (del Sol \& Olave).

Los estudios en cadáveres señalan una baja prevalencia de estas variaciones, del orden del 4\% (Miguel et al.). Sin embargo, los reportes quirúrgicos señalan una incidencia mucho mayor, del 27\% (Merida-Velasco et al.); situación que deja entrever cierta incuria en el noble acto de la disección o el desinterés de comunicar estos hallazgos por parte de los anatomistas. Ignorando si nuestro medio refleja lo indicado en el párrafo precedente, la casuística chilena publicada es pobre, existiendo un reporte, de del Sol \& Olave, quienes encuentran una variación muscular en la fosa axilar, el músculo elevador del tendón del M. latísimo del dorso, en un cadáver de sexo masculino de una serie de 108 cuerpos disecados en la Facultad de Medicina de la Universidad de La Frontera. En nuestro Departamento de Anatomía en 112 cadáveres disecados (desde 1979 a la fecha), 104 hombres y 8 mujeres, hemos detectados dos casos de arco axilar clásicamente descrito por Langer, además del caso presentado en este reporte, todos en sujetos de sexo masculino.

Es interesante notar que el vientre superior de este fascículo muscular, dispuesto sobre el M. subescapular, recibe una fina rama de la arteria axilar; rama supernumeraria que 


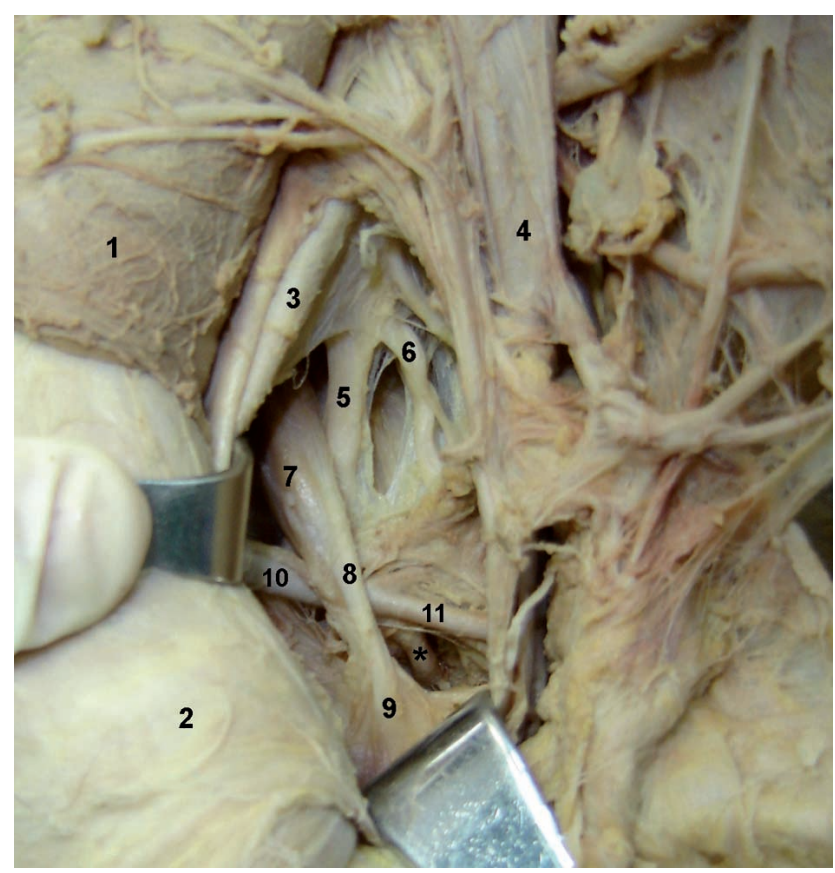

Fig. 1. Disección en el plano profundo de la región axilar derecha. 1. M. pectoral menor; 2. M. pectoral mayor; 3. A. axilar rechazada hacia lateral; 4. V. axilar; 5. N. axilar; 6. N. subescapular inferior; 7. Vientre superior del remanente vestigial del Panniculus carnosus; 8. Tendón intermedio del remanente vestigial; 9. Porción muscular inferior del remanente vestigial; 10. A. subescapular; 11 . A. toracodorsal; * = A. circunfleja escapular.

surge desde la cara posterior de la segunda porción de este vaso. Del mismo modo, por la cara anterior de este vientre muscular discurre un ramo del nervio pectoral lateral que lo inerva, dato que coincide con lo publicado recientemente por Afshar \& Golalipour (2005). Este patrón de inervación, proveniente de ramos primarios ventrales de nervios raquídeos, tal como ocurre con el Panniculus carnosus de mamíferos inferiores, da soporte a la teoría de que estos fascículos detectados ocasionalmente en humanos corresponden a remanentes vestigiales en la fosa axilar de aquel extenso plano muscular (Langworthy; Besana-Ciani \& Greenall).

La literatura señala que la presencia de estos remanentes musculares tiene implicancias clínicas y quirúrgicas, percibiéndose como una masa axilar que puede confundirse con nodos linfáticos infartados o con un tumor de tejidos blandos (Miguel et al.) En especial, el arco axilar clásico se asocia con compresión vascular y linfática que puede provocar trombosis o linfoedema y, en casos extremos, con fenómenos neurovasculares que afectan a ramos terminales del plexo braquial (Merida-Velasco et al.). En la exploración quirúrgica de la axila, la presencia de estas variaciones puede confundir y dificultar el acceso y la exposición de los grupos de linfonodos de la región, situación de particular importancia en la biopsia del nodo centinela (Petrasek et al.). En el caso que aquí se describe, la situación del músculo aberrante complicaría especialmente el acceso al grupo linfático posterior de la axila, ya que toma relación con la porción terminal de la arteria subescapular; además, el tendón intermedio, por su dis-

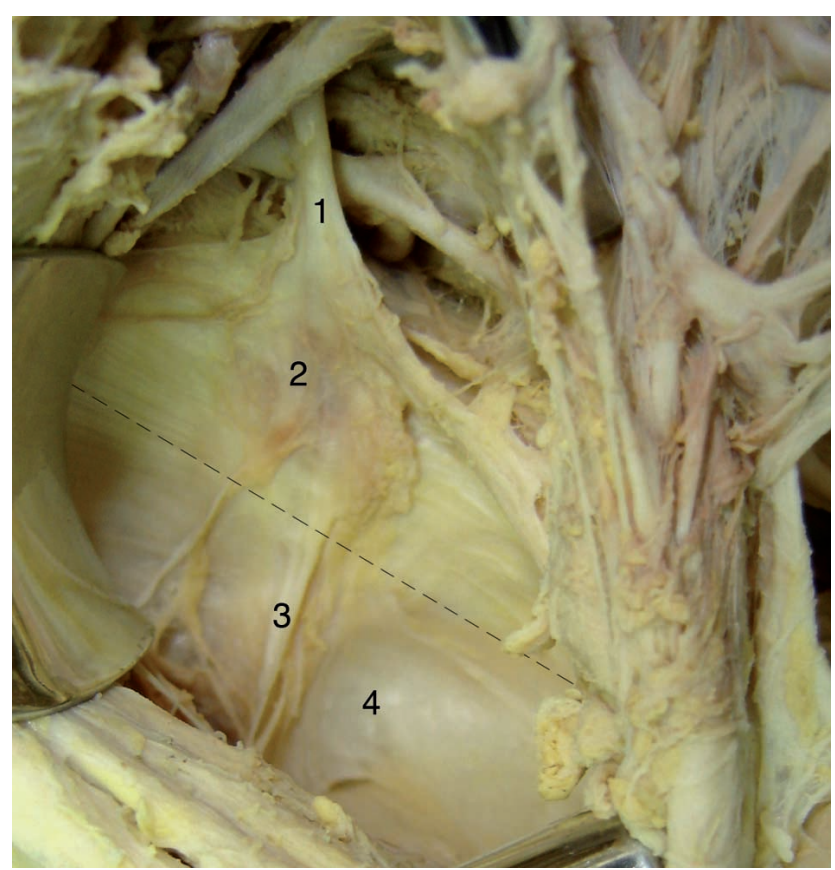

Fig. 2. Disección de la zona baja de la pared posterior de la región axilar derecha. 1. Tendón intermedio del remanente vestigial del Panniculus carnosus; 2. Porción muscular inferior del remanente vestigial; 3. Porción del remanente vestigial que se extiende hacia la fascia de la base de la región axilar. 4. Fascia de la base de la región axilar. La línea punteada indica el límite entre el tendón del M. latisimo del dorso (que se observa por transparencia) y la base de la axila.

posición, puede ser confundido con una rama del fascículo posterior del plexo braquial.

Del Sol \& Olave han publicado recientemente una variación muscular similar a la presentada en este reporte, el "músculo elevador del tendón del músculo latísimo del dorso" el cual, al igual que el caso nuestro, se extiende desde el proceso coracoides hasta el tendón del M. latísimo del dorso. Aún cuando estos autores no entregan datos respecto de las dimensiones del fascículo muscular, en nuestro caso ese nombre nos parece presuntuoso ya que, biomecánicamente, es improbable que el delgado vientre muscular logre levantar a la gruesa masa del tendón del latísimo del dorso. Por su disposición, más bien nos parece que esta variación muscular tensa o eleva la fascia axilar, contrarrestando la acción del M. pectoral menor con su extensión en el ligamento suspensorio de la axila de Gerdy (Testut, 1960), por lo que si nos permitieran la licencia podríamos llamarle "músculo tensor de la fascia axilar".

La presencia de estas variaciones anatómicas, observadas en cadáveres utilizados con fines docentes, son especialmente atractivas para nuestros alumnos y para los docentes y representan un desafío para explicar su génesis. Además, colocan en el tapete, una y otra vez, el tema de las variaciones anatómicas tan tristemente olvidadas en los nuevos textos de anatomía; cobrando cuerpo la sentencia de Sir William Osler: "Variability is the law of life". Esto permite resaltar lo formativo de la disección anatómica, método no superado aún para aprender, realmente, Anatomía Humana (Bravo \& Inzunza, 1995). 
INZUNZA, O.; MARÍN, A.; PINO, F.; NAVARRETE, C. \& VARGAS, A. Panniculus carnosus, remanentes vestigiales en la región axilar. Int. J. Morphol., 26(4):841-844, 2008.

SUMMARY: The anatomical variations of the muscular elements of the axillary region are infrequent and, generally, they are forgotten in modern texts of anatomy. Nevertheless, when they exist, they present a serious obstacle for the surgeon, since they alter the anatomical landmarks of reference for the approach to important neural and vascular elements of the region. Since the 19th century some authors have described a variety of small muscular fascicles originated in the "panniculus carnosus", that are arranged in relation to neurovasculares elements of the axila. In this occasion we present a finding performed in a corpse, male sex, dissected in the Department of Anatomy of the School of Medicine of the Pontificia Universidad Católica de Chile. In the right axillary region, a muscular fascicle is observed that extends from the coracoid process, arranged in front of the subscapularis muscle, and passing behind the neurovascular elements of the axila. In its course, this fascicle takes close relation with the axillary nerve and with the distal portion of the subscapular artery. This aberrant fascicle ends as an aponeurotic expansion, arranged ventral to the tendon of the latissimus dorsi muscle, which melts with the axillary fascia.

KEY WORDS: Anatomy; Axilar region; Panniculus carnosus.

\section{REFERENCIAS BIBLIOGRÁFICAS}

Afshar M, Golalipour M. Inervación del Arco Muscular de la Axila por un Ramo Pectoral. Int. J. Morphol., 23(3):27980, 2005.

Bergman, R.; Afifi, A. \& Miyauchi, R. 2006. Panniculus Carnosus [Web Document]<http://www.anatomyatlases. org/Anatomic Variants/MuscularSystem/Text/P/ 05Panniculus.shtml>.

Besana-Ciani, I. \& Greenall, M. J.Langer`s axillary arch : Anatomy, Embryological Features and Surgical Implications. Surgeon, 3(5):325-327, 2005.

Bravo, H. \& Inzunza, O. Evaluación de Algunos Programas Computacionales en la Enseñanza de la Anatomía y Neuroanatomía de la Facultad de Medicina de la Pontificia Universidad Católica de Chile. Rev. Chil. Anat., 13(1):7986, 1995.

Gruber, W. Neue Anomalien als Beitroege der Menschen. Phys. Chirug. u Path. Anat., 6:31, 1844.

Del Sol, M. \& Olave, E. Elevator Muscle of the Tendon of Latissimus Dorsi Muscle. Clinical Anatomy, 18:112-4, 2005.

Kutiyanawala, M. A.; Stotter, A. \& Windle, R. Anatomical Variants during Axillary Dissection. British J. Surg., 85:393-4, 1998.

Langer, C. Zur Anatomie des Musculus Latissimus Dorsi. Oseterreichische Med Wochenschrift 15:454-58, 1846.

Langworthy, O. The Panniculus Carnosus and Pouch Musculature of the Opossum, a Marsupial. J. of Mammalogy 13(3):241-51, 1932.

Langworthy, O. The Panniculus Carnosus in Cat and Dog and its Genetical Relatoin to the Pectoral Musculature. J. of Mammalogy 5(1):49-63, 1924.
Merida-Velasco, J. R.; Rodriguez Vasquez, J. F.; MeridaVelasco, J. A.; Sobrado Perez, J. \& Collado, J. J. Axillary Arch: Potential Cause of Neurovascular Compression Syndrome. Clin. Anat., 16:514-9, 2003.

Miguel, M.; Lusá, M.; Ortiz, J. C.; Porta, N.; Lorente, M. \& Götzens, V. The Axillopectoral Muscle (of Langer) Report of Three Cases. Surg. Radiol. Anat., 23:341-3, 2001.

Petrasek, A. J.; Semple, J. L. \& McCready, D. R. The Surgical and Oncologic Significance of the Axillary Arch During Axillary Lynphadenectomy. Can. J. Surg., 40(1):44-47.

Ramsay, A. Citado por Besana-Ciani I, Greenall M. J. 2005. in Surgeon, 3(5):325-7, 1975.

Ramsay, A. Account of Unusual Conformations of Some Muscles and Vessels. Edinburgh Med. Surg. J., 8:281-3, 1812.

Ruge, G. Die Hautmusculatur der Monotremen und ihre Beziehungen zu dem Marsupial und Mammarapparate. Aus Semon, Zoolog Forschungreisen, ii. Jenaische Denkschriften v. 1895.

Testut, L. \& Latarjet A. Tratado de Anatomía Humana. Madrid, Salvat Editores, 1960

Tillaux, P. Tratado de Anatomía Topográfica. Madrid, Biblioteca Ilustrada, 1880.

Wood, J.Variations in Human Myology Observed During the Winter Session of 1867-1868 at King's College, London. Proc. R. Soc. London, 16:483-525, 1868.

Dirección para correspondencia:

Prof. Dr. Oscar Inzunza

Departamento de Anatomía Normal

Escuela de Medicina,

Pontificia Universidad Católica de Chile

CHILE

Email: oinzunza@med.puc.cl_Recibido : 22-08-2008

Aceptado: $27-09-2008$ 